Pacific Journal of Mathematics

PERMUTATIONS, MATRICES, AND GENERALIZED YOUNG 


\title{
PERMUTATIONS, MATRICES, AND GENERALIZED YOUNG TABLEAUX
}

\author{
DONALD E. KNUTH
}

\begin{abstract}
A generalized Young tableau of "shape" $\left(p_{1}, p_{2}, \cdots, p_{m}\right)$, where $p_{1} \geqq p_{2} \geqq \cdots \geqq p_{m} \geqq 1$, is an array $Y$ of positive integers $y_{i j}$, for $\mathbf{1} \leqq j \leqq p_{i}, \mathbf{1} \leqq i \leqq m$, having monotonically nondecreasing rows and strictly increasing columns. By extending a construction due to Robinson and Schensted, it is possible to obtain a one-to-one correspondence between $m \times n$ matrices $A$ of nonnegative integers and ordered pairs $(P, Q)$ of generalized Young tableaux, where $P$ and $Q$ have the same shape, the integer $i$ occurs exactly $a_{i 1}+\cdots+a_{i n}$ times in $Q$, and the integer $j$ occurs exactly $a_{1 j}+\cdots+a_{m j}$ times in $P$. A similar correspondence can be given for the case that $A$ is a matrix of zeros and ones, and the shape of $Q$ is the transpose of the shape of $P$.
\end{abstract}

Figure 1 shows two arrangements of integers which we will call generalized Young tableaux of shape $(6,4,4,1)$. A generalized Young tableau of shape $\left(p_{1}, p_{2}, \cdots, p_{m}\right)$ is an array of $p_{1}+p_{2}+\cdots p_{m}$ positive integers into $m$ left-justified rows, with $p_{i}$ elements in row $i$, where $p_{1} \geqq p_{2} \geqq \cdots \geqq p_{m}$; the numbers in each row are in nondecreasing order from left to right, and the numbers in each column are in strictly increasing order from top to bottom. (The special case where the elements are the integers $1,2, \cdots, N=p_{1}+p_{2}+\cdots+p_{m}$, each used exactly once, was introduced by Alfred Young in 1900 as an aid in the study of irreducible representations of the symmetric group on $N$ letters; see [6].)

Consider on the other hand the $6 \times 7$ array

$$
A=\left(\begin{array}{lllllll}
0 & 0 & 1 & 0 & 0 & 0 & 0 \\
0 & 0 & 0 & 0 & 0 & 2 & 0 \\
1 & 1 & 1 & 1 & 0 & 1 & 0 \\
0 & 0 & 1 & 0 & 1 & 0 & 0 \\
2 & 1 & 0 & 1 & 0 & 0 & 0 \\
0 & 0 & 0 & 0 & 0 & 0 & 1
\end{array}\right)
$$

having respective column sums $\left(c_{1}, \cdots, c_{7}\right)=(3,2,3,2,1,3,1)$ and row sums $\left(r_{1}, \cdots, r_{6}\right)=(1,2,5,2,4,1)$. Note that in Figure 1 the integer $i$ occurs $r_{i}$ times in $Q$, and the integer $j$ occurs $c_{j}$ times in $P$. In this paper we shall give a constructive procedure which yields a one-to-one correspondence between matrices $A$ of nonnegative integers. 


$P$
\begin{tabular}{|l|l|l|l|l|l|l|l|l|l|l|l|}
\hline 1 & 1 & 1 & 2 & 4 & 7 & 1 & 2 & 2 & 3 & 3 & 6 \\
\hline 2 & 3 & 3 & 5 & & & 3 & 3 & 3 & 4 & \\
\hline 3 & 4 & 6 & 6 & & & & \\
\hline 6 & & & 5 & 5 & 5 & \\
\hline
\end{tabular}

FIGURE 1.

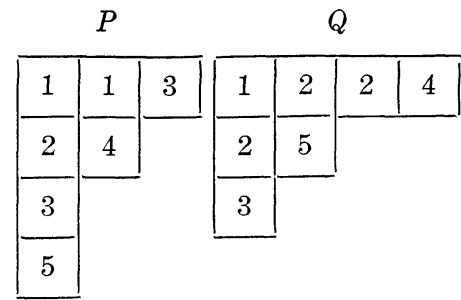

FIGURE 2.

and ordered pairs of equal-shape generalized Young tableaux $(P, Q)$ such that the row and column sums of $A$ correspond in the same manner to the number of occurrences of elements in $P$ and $Q$. In particular, our procedure shows how to construct (1.1) from Figure 1 and conversely.

Figure 2 shows two generalized Young tableaux whose shapes are transposes of each other. A modification of the first construction leads to another procedure which gives a similar correspondence between zero-one matrices $A$ and such pairs of tableaux. For example, the second construction associates the matrix

$$
A=\left(\begin{array}{lllll}
0 & 0 & 1 & 0 & 0 \\
1 & 1 & 0 & 1 & 0 \\
1 & 0 & 0 & 0 & 0 \\
0 & 0 & 0 & 0 & 1 \\
0 & 0 & 1 & 0 & 0
\end{array}\right)
$$

with Figure 2. When the column sums of $A$ are all $\leqq 1$, the two constructions are essentially identical, differing only in that $P$ is transposed.

Matrices $A$ of nonnegative integers correspond in an obvious way to two-line arrays of positive integers

$$
\left(\begin{array}{llll}
u_{1} & u_{2} & \cdots & u_{N} \\
v_{1} & v_{2} & \cdots & v_{N}
\end{array}\right)
$$

where the pairs $\left(u_{k}, v_{k}\right)$ are arranged in nondecreasing lexicographic order from left to right, and where there are exactly $a_{i j}$ occurrences of the pair $(i, j)$. For example, the matrix (1.1) corresponds in this way to

$$
\left(\begin{array}{lllllllllllllll}
1 & 2 & 2 & 3 & 3 & 3 & 3 & 3 & 4 & 4 & 5 & 5 & 5 & 5 & 6 \\
3 & 6 & 6 & 1 & 2 & 3 & 4 & 6 & 3 & 5 & 1 & 1 & 2 & 4 & 7
\end{array}\right) .
$$

Such two-line arrays can be regarded as generalized permutations, for when $A$ is a permutation matrix the corresponding two-line array is 
the permutation corresponding to $A$. When $A$ is a zero-one matrix, the pairs $\left(u_{k}, v_{k}\right)$ in (1.3) are all distinct.

Our construction works with two-line arrays (1.3) instead of the original matrices (although it is, of course, possible to translate everything we do into the matrix notation). The special case where $u_{k}=$ $k, 1 \leqq k \leqq N$, was treated by Craige Schensted in 1961 [7]; in this case $A$ is a zero-one matrix with $N$ rows, each row-sum being equal to unity. Our first construction is identical to Schensted's in this particular case. Another procedure which can be shown to be essentially equivalent to Schensted's construction was published already in 1938 by Gilbert de B. Robinson [5, Sec. 5], although he described the algorithm rather vaguely and in quite different terms.

Section 2 below presents Schensted's algorithm in detail, and $\S 3$ uses that algorithm to achieve the first correspondence. A graphtheoretical interpretation of the correspondence, given in $\S 4$, allows us to conclude that transposition of the matrix $A$ corresponds to interchanging $P$ and $Q$; hence we obtain a useful one-to-one correspondence between symmetric matrices $A$ and (single) generalized Young tableaux.

Section 5 shows how to modify the preceding algorithms to obtain the second correspondence. Finally in $\S 6$ a combinatorial characterization is given of all matrices having a given value of $P$; this leads to an "algebra of tableaux."

As a consequence of the algorithms in this paper it is possible to obtain a constructive proof of MacMahon's classical formulas for the enumeration of plane partitions, as well as new enumeration formulas for certain rather general kinds of plane partitions. These applications will be reported elsewhere [1].

2. The insertion and deletion procedures. It is convenient to regard a generalized Young tableau of shape $\left(p_{1}, p_{2}, \cdots, p_{m}\right)$ as a doubly-infinite array

$$
Y=\begin{array}{lllll}
y_{00} & y_{01} & y_{02} & y_{03} & \cdots \\
y_{10} & y_{11} & y_{12} & y_{13} & \cdots \\
y_{20} & y_{21} & y_{22} & y_{23} & \cdots
\end{array}
$$

with $y_{i j}=0$ if $i$ or $j$ is zero, $y_{i j}=\infty$ if $i>m$ or $j<p_{\imath}$, and $0<$ $y_{i j} \neq \infty$ when $1 \leqq i \leqq m, 1 \leqq j \leqq p_{i}$. We simply border the tableau with zeros at the top and left, and we put os symbols elsewhere. Using the further convention

this doubly-infinite array satisfies the inequalities 


$$
\begin{gathered}
y_{i j} \leqq y_{i(j+1)}, \quad y_{i j}<y_{(i+1) j}, \\
\text { for all } i, j \geqq 0
\end{gathered}
$$

Convention (2.2) may appear somewhat strange, but it does not make the transitive law invalid, and it is a decided convenience in what follows because of the uniform conditions (2.3).

We can now describe Schensted's procedure for inserting a new positive integer $x$ into a generalized Young tableau $Y$. The following algorithm contains parenthesized "assertions" about the present state of affairs, each of which is easily verified from previously verified assertions; hence we are presenting a proof of the validity of the algorithm at the same time as the algorithm itself is being presented. (Cf. [2, pp. 2-3, 15-16].)

\section{INSERT $(x)$ :}

I1. Set $i \leftarrow 1$, set $x_{1} \leftarrow x$, and set $j$ to some value such that $y_{1 j}=\infty$.

I2. (Now $y_{(i-1) j}<x_{i}<y_{i j}$, and $x_{i} \neq \infty$.) If $x_{i}<y_{i(j-1)}$, decrease $j$ by 1 and repeat this step. Otherwise set $x_{i+1} \leftarrow y_{i j}$ and set $r_{i} \leftarrow j$.

I3. (Now $y_{i(j-1)} \leqq x_{i}<x_{i+1}=y_{i j} \leqq y_{i(j+1)}, y_{(i-1) j}<x_{i}<x_{i+1}=y_{i j}<$ $y_{(i+1) j}, r_{i}=j$, and $x_{i} \neq \infty$.) Set $y_{i j} \leftarrow x_{i}$.

I4. (Now $y_{i(j-1)} \leqq y_{i j}=x_{i}<x_{i+1} \leqq y_{i(j+1)}, y_{(i-1) j}<y_{i j}=x_{i}<x_{i+1}<$ $y_{(i+1) j}, r_{i}=j$, and $x_{i} \neq \infty$.) If $x_{i+1} \neq \infty$, increase $i$ by 1 and return to step I2.

I5. Set $s \leftarrow i$ and $t \leftarrow j$, and terminate the algorithm. (Now the conditions

$$
y_{s t} \neq \infty, x_{s+1}=y_{s(t+1)}=y_{(s+1) t}=\infty
$$

hold.)

The parenthesized assertions in steps I3 and I4 serve to verify that $Y$ remains a generalized Young tableau throughout the algorithm. The algorithm always terminates in finitely many steps, since $Y$ contains only finitely many positive integers. The procedure not only inserts $x$ into the tableau, it also constructs two sequences of positive: integers

$$
\begin{gathered}
x=x_{1}<x_{2}<\cdots<x_{s} \\
r_{1} \geqq r_{2} \geqq \cdots \geqq r_{s}=t,
\end{gathered}
$$

where $s$ and $t$ are the quantities specified in the last step of the algorithm.

As an example of this insertion process, let us insert $x=3$ into. the tableau 


$$
Y=\begin{array}{lllll}
1 & 3 & 3 & 5 & 8 \\
2 & 4 & 6 & 6 \\
3 & 5 & 8 \\
4 & &
\end{array}
$$

The algorithm computes $x_{1}=3, r_{1}=4, x_{2}=5, r_{2}=3, x_{3}=6, r_{3}=3$, $x_{4}=8, r_{4}=2, x_{5}=\infty, s=4, t=2$; the tableau has been changed to

$$
Y=\begin{array}{lllll}
1 & 3 & 3 & 3 & 8 \\
2 & 4 & 5 & 6 \\
3 & 5 & 6 \\
4 & 8 &
\end{array}
$$

The input value, 3, "bumped" a 5 from the first row into the second row, where it bumped a 6 to row 3 , etc.

The most important property of Schensted's insertion algorithm is that it has an inverse; we can restore $Y$ to its original condition again, given the values of $s$ and $t$.

DELETE $(s, t)$ :

D1. Set $j \leftarrow t, i \leftarrow s, x_{s+1} \leftarrow \infty$.

D2. (Now $y_{i j}<x_{i+1}<y_{(i+1) j}$ and $y_{i j} \neq \infty$.) If $y_{i(j+1)}<x_{i+1}$ and $y_{i(j+1)} \neq \infty$, increase $j$ by 1 and repeat this step. Otherwise set $x_{i} \leftarrow$ $y_{i j}$ and $r_{i} \leftarrow j$.

D3. (Now $y_{i(j-1)} \leqq y_{i j}=x_{i}<x_{i+1} \leqq y_{i(j+1)}, y_{(i-1) j}<y_{i j}=x_{i}<x_{i+1}<$ $y_{(i+1) j}, r_{i}=j$, and $x_{i} \neq \infty_{\text {.) }}$ Set $y_{i j} \leftarrow x_{i+1}$.

D4. (Now $y_{i(j-1)} \leqq x_{i}<x_{i+1}=y_{i j} \leqq y_{i(j+1)}, y_{(i-1) j}<x_{i}<x_{i+1}=y_{i j}<$ $y_{(i+1) j}, r_{i}=j$, and $x_{i} \neq \infty$.) If $i \neq 1$, decrease $i$ by 1 and return to step D2.

D5. Set $x \leftarrow x_{1}$, and terminate the algorithm. (Now $x \neq \infty$.)

This algorithm obviously terminates, since $Y$ contains only finitely many positive integers. The parenthesized assertions in steps D3 and D4 show that $Y$ remains a generalized Young tableau; moreover, these assertions uniquely define the value of $j$, and they are precisely the same as those of steps I4 and I3, respectively. Hence the deletion algorithm recomputes the sequences (2.5) determined by the insertion algorithm, and it restores $Y$ to its original condition. The reader may verify, for example, that DELETE $(4,2)$ transforms $(2.7)$ into $(2.6)$.

Conversely, if we start with any generalized Young tableau, $Y$, and if we choose two integers $(s, t)$ such that $(2.4)$ holds, the procedure DELETE $(s, t)$ will specify some positive integer $x$ in step D5, and $x$ is removed from the tableau; the subsequent operation INSERT $(x)$ 
will put $x$ back again, recompute $s$ and $t$, and restore $Y$ to its original form. Thus INSERT and DELETE are inverses of each other.

We will now establish an important property relating the quantities $x, s, t$ in successive insertions (cf. Schützenberger [8, Remarque 2]).

THEOREM 1. Let $x, x^{\prime}$ bo positive integers. If INSERT $(x)$, determining $s$ and $t$, is immediately followed by INSERT $\left(x^{\prime}\right)$, determining $s^{\prime}$ and $t^{\prime}$, then

$$
\begin{array}{r}
x \leqq x^{\prime} \text { if and only if } s \geqq s^{\prime} \\
\text { if and only if } t^{\prime}>t .
\end{array}
$$

Proof. (a) We prove first that

$$
x \leqq x^{\prime} \text { implies } s \geqq s^{\prime} \text { and } t^{\prime}>t .
$$

Let the sequences $(2.5)$ be denoted by $x_{i}, r_{i}(1 \leqq i \leqq s)$ and $x_{i}^{\prime}, r_{i}^{\prime}(1 \leqq$ $i \leqq s^{\prime}$ ) when $x$ and $x^{\prime}$ are respectively inserted. Assume by induction that $s \geqq i$ and $s^{\prime} \geqq i$ and $x_{i} \leqq x_{i}^{\prime}$ (this holds initially for $i=1$ ). Consider the state of affairs at the beginning of step I3, when $x_{i}^{\prime}$ is about to be inserted. We have $x_{i}=y_{i j}$ for $j=r_{i}$, hence $j^{\prime}=r_{i}^{\prime}>j$; it follows that $x_{i+1} \leqq y_{i(j+1)} \leqq y_{i j^{\prime}}=x_{i+1}^{\prime}$. If $s^{\prime}=i$ then $s \geqq s^{\prime}$ and $t^{\prime}=$ $j^{\prime}>j \geqq t$, so (2.9) holds. On the other hand if $s^{\prime}>i$ then $x_{i+1}^{\prime} \neq \infty$, hence $x_{i+1} \neq \infty$, so $s>i$ and the inductive hypothesis is valid for $i$ replaced by $i+1$.

(b) The theorem now follows if we can prove that

$$
x^{\prime}<x \text { implies } s^{\prime}>s \text { and } t \geqq t^{\prime} .
$$

The proof is like part (a), but just different enough to require care. Assume by induction that $s \geqq i$ and $s^{\prime} \geqq i$ and $x_{i}^{\prime}<x_{i}$ (this holds initially for $i=1$ ). Consider the state of affairs when $x_{i}^{\prime}$ is about to be inserted, as in part (a); we have $j \geqq r_{i}^{\prime}=j^{\prime}$, hence $x_{\imath+1}^{\prime}=y_{i j^{\prime}} \leqq$ $x_{i}<x_{i+1}$. In particular, $x_{i+1}^{\prime} \neq \infty$, so $s^{\prime}>i$. If $s=i$ then $t=j \geqq$ $j^{\prime} \geqq t^{\prime}$, so (2.10) holds. If $s>i$ then the induction hypothesis is valid for $i$ replaced by $i+1$.

3. A one-to-one correspondence. We are now ready to give a fairly direct correspondence between two-line arrays of positive integers

$$
\left(\begin{array}{llll}
u_{1} & u_{2} & \cdots & u_{N} \\
v_{1} & v_{2} & \cdots & v_{N}
\end{array}\right)
$$

where the pairs $\left(u_{k}, v_{k}\right)$ are arranged in nondecreasing lexicographic order from left to right, and ordered pairs $(P, Q)$ of generalized Young tableaux having the same shape, where the elements of $P$ are $v_{1}, v_{2}, \cdots, v_{N}$ 
and the elements of $Q$ are $u_{1}, u_{2}, \cdots, u_{N}$.

The procedure, which we will call construction $A$, starts with s empty" tableaux :

$$
\begin{aligned}
& p_{00}=p_{0 j}=p_{i 0}=0, p_{i j}=\infty ; \\
& q_{00}=q_{0 j}=q_{i 0}=0, q_{i j}=\infty,
\end{aligned}
$$

for all $i, j \geqq 1$.

Then we do the following steps for $k=1,2, \cdots, N$ (in this order):

A1. INSERT $\left(v_{k}\right)$ into tableau $P$, determining values $s_{k}, t_{k}$ in step I5.

\section{A2. Set $q_{s_{k} t_{k}} \leftarrow u_{k}$.}

The reader may verify, for example, that this procedure takes the two-line array (1.4) into the tableaux of Figure 1. It is clear from the construction that $P$ and $Q$ have the same shape, since the insertion procedure removes the $\infty$ from row $s$ and column $t$ of the tableau. Furthermore, since $u_{1} \leqq u_{2} \leqq \cdots \leqq u_{N}$, and since step A2 inserts an element on the 'periphery' of $Q$, it is clear that $Q$ will be a generalized Young tableau if we can verify that no equal elements fall into the same column of $Q$. The latter property follows immediately from Theorem 1, for $u_{k}=u_{k+1}$ implies that $v_{k} \leqq v_{k+1}$, hence $t_{k+1}>t_{k}$.

The inverse construction, which we will call construction $B$, starts with two generalized Young tableaux, $P$ and $Q$, of shape $\left(p_{1}, p_{2}, \cdots\right.$, $p_{m}$ ); let $N=p_{1}+p_{2}+\cdots+p_{m}$ be the total number of elements. We do the following steps for $k=N, \cdots, 2,1$ (in this order):

B1. Find $s_{k}, t_{k}$ such that $q_{s_{k} t_{k}}$ is the largest positive integer element of $Q$, where $t_{k}$ is as large as possible. Set $u_{k} \leftarrow q_{s_{k} t_{k}}$ and then set $q_{s_{k} t_{k}} \leftarrow \infty$.

B2. DELETE $\left(s_{k}, t_{k}\right)$ from tableau $P$, determing a value $x$ in step D5; set $v_{k} \leftarrow x$.

The algorithm clearly reverses construction $A$. Conversely, if we apply construction $B$ to any given pair of generalized Young tableaux having the same shape, we can see by Theorem 1 that the pairs $\left(u_{1}\right.$, $\left.v_{1}\right),\left(u_{2}, v_{2}\right), \cdots,\left(u_{N}, v_{N}\right)$ are in lexicographic order, i.e., that $u_{1} \leqq$ $u_{2} \leqq \cdots \leqq u_{N}$ and that $u_{k}=u_{k+1}$ implies $v_{k} \leqq v_{k+1}$. It follows readily that construction $A$ reverses construction $B$, hence the one-to-one correspondence is established.

Theorem 2. Constructions $A$ and $B$, which are inverses of each other, establish a ons-to-one correspondence botween two-line arrays and generalized Young tableaux having the properties stated above. 
By the previously mentioned correspondence between two-line arrays. and matrices of nonnegative integers, we have therefore verified the first result advertised in $\S 1$.

4. A graph-theoretical viewpoint. The correspondence in the preceding section can be looked at in another way, if we try to build the $P$ and $Q$ tableaux one row at a time instead of using the insertion procedure. The first rows of $P$ and $Q$ can be interpreted in terms of a certain labelled directed graph, which might be called the "inversion digraph" $D_{1}$ of the given generalized permutation (3.1); similarly the second rows of $P$ and $Q$ are related to the "second-order inversion. digraph" $D_{2}$ derived from $D_{1}$, and so on. We will now study this. graph-theoretical interpretation of Schensted's construction, in order to. deduce further properties of the correspondence.

Given a two-line array

$$
\left(\begin{array}{llll}
u_{1} & u_{2} & \cdots & u_{N} \\
v_{1} & v_{2} & \cdots & v_{N}
\end{array}\right)
$$

we construct its corresponding "inversion digraph" $D_{1}$ as follows: There are $N$ vertices, to which the respective labels $\left(u_{1}, v_{1}\right),\left(u_{2}, v_{2}\right), \cdots$, $\left(u_{N}, v_{N}\right)$ are attached; in the discussion below, when we refer to a vertex $(u, v)$ we mean any one of the vertices whose label is $(u, v)$. An arc passes from vertex $(u, v)$ to vertex $\left(u^{\prime}, v^{\prime}\right)$, when $(u, v) \neq\left(u^{\prime}, v^{\prime}\right)$, if and only if

$$
u \leqq u^{\prime} \text { and } v \leqq v^{\prime}
$$

Furthermore we construct arcs between vertices with identical labels: by putting all vertices with given label $(u, v)$ into some arbitrary order, say $V_{1}, V_{2}, \cdots, V_{k}$, and drawing ares from $V_{i}$ to $V_{j}$ if and only if $i<j$. For example, Figure 3 shows the inversion digraph corresponding to

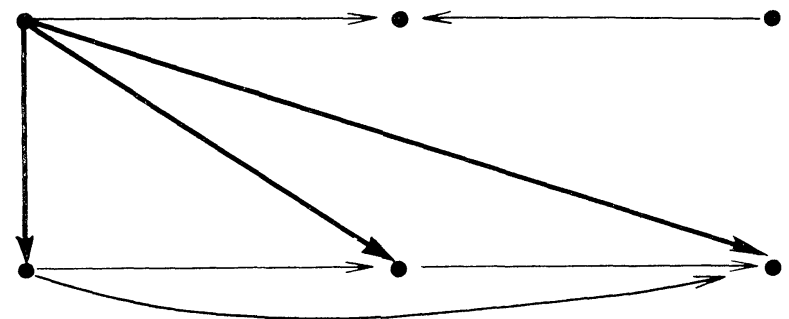

$(1,3)$

Figure 3. 


$$
\left(\begin{array}{llllll}
1 & 1 & 1 & 1 & 2 & 3 \\
2 & 3 & 3 & 3 & 1 & 2
\end{array}\right)
$$

When the two-line array is a permutation of the integers $\{1,2, \cdots$, $N\}$, the number of arcs in $D_{1}$ is equal to $\left(\begin{array}{l}n \\ 2\end{array}\right)$ minus the number of inversions in the permutation according to the classical theory, hence the name "inversion digraph." It is easy to see in the general case that $D_{1}$ has no oriented cycles, and in fact it is the digraph of a partial ordering. Note that our definition of $D_{1}$ does not require that the pairs $\left(u_{k}, v_{k}\right)$ of $(4.1)$ be in lexicographic order from left to right; only the pairs themselves are used. Furthermore (4.2) is symmetric in $u$ and $v$, hence the inversion digraph of

$$
\left(\begin{array}{llll}
v_{1} & v_{2} & \cdots & v_{N} \\
u_{1} & u_{2} & \cdots & u_{N}
\end{array}\right)
$$

is isomorphic to the inversion digraph of (4.1). This observation will be important to us later.

We now partition the vertices of $D_{1}$ into disjoint classes $C_{1}, C_{2}, \cdots$, as follows: $C_{1}$ contains the "source" vertices, i.e., those with no arcs leading in to them; and for $l \geqq 1, C_{l+1}$ consists of all vertices which are sources when the vertices of $C_{1} \cup \cdots \cup C_{l}$ (and all arcs touching them) are removed. For example in Figure 3 we have

$$
\begin{aligned}
& C_{1}=\{(1,2),(2,1)\}, \\
& C_{2}=\{(1,3),(3,2)\}, \\
& C_{3}=\{(1,3)\}, \\
& C_{4}=\{(1,3)\} .
\end{aligned}
$$

(The vertices denoted by $(1,3)$ in $C_{2}, C_{3}, C_{4}$ are actually distinct, because of our conventions for dealing with vertices having equal labels.) The reader may easily verify that, in general, $C_{l}$ consists of all vertices $V$ such that the longest path to $V$ in $D_{1}$ has length $l-1$. This partitioning is closely related to the well-known procedure for "topological sorting" [2, pp. 258-268].

If $(u, v)$ and $\left(u^{\prime}, v^{\prime}\right)$ are distinct vertices of the same class $C_{l}$, there is no arc joining them; it follows from the construction that $u \neq u^{\prime}$ and $v \neq v^{\prime}$. Furthermore if $u<u^{\prime}$ then $v^{\prime}<v$, and conversely ; therefore we can arrange the vertices of $C_{l}$ into the following order:

$$
C_{l}=\left(u_{l 1}, v_{l 1}\right),\left(u_{l 2}, v_{l 2}\right), \cdots,\left(u_{l n_{l}}, v_{l n_{l}}\right)
$$

where

$$
\begin{aligned}
& u_{l 1}<u_{l 2}<\cdots<u_{l n l} \\
& v_{l 1}>v_{l 2}>\cdots>v_{l n l} .
\end{aligned}
$$


Lemma 1. The digraph $D_{1}$ constructed above has the following relation to the correspondence defined in § 3: If the nonempty verten classes are $C_{1}, \cdots, C_{d}$, the first row of $P$ at the end of the construction is

$$
v_{1 n_{1}}, v_{2 n_{2}}, \cdots, v_{d n_{d}}
$$

(in the notation of (4.5)), and the first row of $Q$ is

$$
u_{11}, u_{21}, \cdots, u_{d 1} \text {. }
$$

Moreover if we denote the two sequences (2.5) obtained during the operation INSERT $\left(v_{k}\right)$ by

$$
\begin{aligned}
v_{k}=x_{k 1} & <x_{k 2}<\cdots<x_{k s_{k}}, \\
r_{k 1} & \geqq r_{k 2} \geqq \cdots \geqq r_{k s_{k}}=t_{k},
\end{aligned}
$$

then the pair $\left(u_{k}, v_{k}\right)$ of (3.1) belongs to class $C_{l}$ if and only if $l=r_{k 1}$.

Proof. We want to show that the vertices of class $l$ are those pairs $\left(u_{k}, v_{k}\right)$ which affect the $l$-th element of the first row of $P$ during the insertion process. The proof is easily carried out by induction on $N$; for if we add a new vertex $\left(u_{N+1}, v_{N+1}\right)$ which is lexicographically greater than all other vertices of $D_{1}$, no new arcs lead from this vertex, while there are arcs leading from a vertex of class $C_{l}$ to this new vertex if and only if $v_{l n_{l}} \leqq v_{N+1}$.

COROLLARY (Schensted). The number of columns in the generalized Young tableaux $P, Q$ corresponding to (3.1) is the length of the longest nondecreasing subsequence of the sequence $v_{1}, v_{2}, \cdots, v_{N}$.

Proof. We have observed that $C_{d}$ is nonempty if and only if there is a path of length $d-1$ in $D_{1}$; such a path corresponds to a nondecreasing subsequence of length $d$.

We now have characterized the first rows of $P$ and $Q$ in terms of the labelled digraph $D_{1}$. Since Schensted's construction behaves on row $(i+1)$ in essentially the same way as it does on row $i$ (inserting elements that were bumped down from row $i$ ), we can see how to characterize the remaining rows of $P$ and $Q$. Assuming that the $i$-th order inversion digraph $D_{i}$ has been defined, we will construct $D_{i+1}$ by leaving out one vertex of each class and by changing the labels. If class $C_{l}$ of $D_{i}$ is given by (4.4) and (4.5), we include $n_{l}-1$ vertices labelled

$$
\left(u_{l 2}, v_{l 1}\right),\left(u_{l 3}, v_{l 2}\right), \cdots,\left(u_{l n_{l}}, v_{l\left(n_{l-1}\right)}\right)
$$


in $D_{i+1}$. After the vertices of $D_{i+1}$ have been determined in this way, from all classes of $D_{i}$, the arcs of $D_{i+1}$ are defined in precisely the same manner of we have defined the arcs of $D_{i}$.

The vertex labels of $D_{i+1}$ correspond to a two-line array. A few moments' reflection will show that, in view of Lemma 1, construction $A$ in $\S 3$ builds rows $2,3, \ldots$ of $P$ and $Q$ in precisely the same way as it would build rows $1,2, \ldots$ if it were given the two-line array corresponding to $D_{2}$ instead of the original two-line array. Hence the second rows of $P$ and $Q$ are respectively given by (4.6) and (4.7) corresponding to $D_{2}$, and in general the digraph $D_{i}$ corresponds to the $i$-th rows of $P$ and $Q$ as $D_{1}$ corresponds to their first rows. This leads to the following result.

THEOREM 3. If the nonnegative integer matrix $A$ corresponds to the generalized Young tableaux $(P, Q)$, then the transposed matrix $A^{T}$ corresponds to $(Q, P)$.

Proof. The two-line array corresponding to $A^{T}$ is obtained from (3.1) by interchanging the two lines and rearranging the columns in lexicographic order. (Cf. (4.3).) We have observed that the resulting graph $D_{1}^{T}$ is isomorphic to $D_{1}$; this isomorphism associates vertex $(v, u)$ of $D_{1}^{T}$ with vertex $(u, v)$ of $D_{1}$. The construction of $D_{i+1}$ from $D_{i}$ shows that the same isomorphism relates $D_{i}^{T}$ to $D_{i}$, hence the theorem follows from Lemma 1.

For the special case of permutation matrices, Robinson [5, p. 755] essentially stated Theorem 3 without proof; a proof was given by Schützenberger [8].

THEOREM 4. The construction of $\S 3$ yields a one-to-one correspondence between symmetric matrices $A$ of nonnegative integers, having respective column sums $\left(c_{1}, c_{2}, \cdots, c_{n}\right)$, and generalized Young tableaux $P$, having $c_{i}$ occurrences of the integer $i$. In this correspondence the number of columns of $P$ having an odd length is the trace of $A$.

Proof. Since $A$ is symmetric, $A=A^{T}$, hence by Theorem $3 P=$ $Q$ in the correspondence. It remains only to verify the connection (suggested by Schützenberger) between odd-length columns and the trace of $A$.

When $A=A^{T}$, there is a corresponding symmetry in the digraph $D_{1}$ since the vertex $(u, v)$ occurs as often as the vertex $(v, u)$. The automorphism $(u, v) \leftrightarrow(v, u)$ shows that each class $C_{l}$ has the form 


$$
\begin{gathered}
u_{l}=\left(u_{l 1}, u_{l n_{l}}\right),\left(u_{l 2}, u_{l\left(n_{l}-1\right)}\right), \cdots,\left(u_{l n_{l}}, u_{l 1}\right), \\
u_{l 1}<u_{l 2}<\cdots<u_{l n_{l}} .
\end{gathered}
$$

(Cf. (4.5).) Hence $C_{l}$ contains 0 or 1 elements of the form $(u, u)$ according as $n_{l}$ is even or odd; and so trace $(A)$ is the number of classes in which $n_{l}$ is odd. Furthermore the graph $D_{2}$ contains as many vertices of the form $(u, u)$ as the number of classes in which $n_{l}$ is even (cf. (4.9) and (4.10)); hence it corresponds to a symmetric matrix $A_{2}$ such that trace $(A)+\operatorname{trace}\left(A_{2}\right)=d=$ number of nonempty classes of $D_{1}=$ number of columns of $P$. Let $P_{2}$ be $P$ with its first row removed; by induction on the number of rows of $P$, we know that $P_{2}$ has trace $\left(A_{2}\right)$ odd columns, hence $P$ has $d$ - trace $\left(A_{2}\right)=\operatorname{trace}(A)$ odd columns.

5. A dual correspondence. Let us say a "dual tableau" is an arrangement of positive integers which is like a generalized Young tableau except that the rows (instead of the columns) are required to have distinct elements. Thus, every dual tableau is the transpose of a generalized Young tableau and conversely.

If $Y$ is a dual tableau, we can insert a new element $x$ into it using a procedure almost identical to Schensted's construction of $\S 2$. The algorithm INSERT ${ }^{*}(x)$ may be defined to be the same as INSERT $(x)$, except that the signs $<$ and $\leqq$ are interchanged throughout the latter algorithm. (An element now bumps down another element equal to itself.) Similarly we obtain an algorithm $\operatorname{DELETE}^{*}(s, t)$ by changing DELETE $(s, t)$ in the same way. The reader may readily verify that, as before, $\operatorname{INSERT}^{*}(x)$ and $\operatorname{DELETE}^{*}(s, t)$ are inverse to each other, and that $Y$ remains a dual tableau throughout each algorithm. We also have

THEOREM 1*. Let $x, x^{\prime}$ be positive integers. If $\operatorname{INSERT}^{*}(x)$, determining $s$ and $t$, is immediately followed by INSERT* ${ }^{*}\left(x^{\prime}\right)$, determining $s^{\prime}$ and $t^{\prime}$, then

$$
\begin{array}{r}
x<x^{\prime} \text { if and only if } s \geqq s^{\prime} \\
\text { if and only if } t^{\prime}>t .
\end{array}
$$

Proof. In the proof of Theorem 1, change the symbol $<$ to $\leqq$ wherever it appears; and change $\leqq$ to $<$, except in the two instances " $y_{i(j+1)} \leqq y_{i j^{\prime}}$ " and " $y_{i j^{\prime}} \leqq x_{i}$ " where the $\leqq$ is to be retained. (Do not change the symbols $>$ and $\geqq$, which have been used consistently for indices instead of elements.) The result is a proof of Theorem 1*.

Now consider a two-line array of positive integers 


$$
\left(\begin{array}{llll}
u_{1} & u_{2} & \cdots & u_{N} \\
v_{1} & v_{2} & \cdots & v_{N}
\end{array}\right)
$$

where the pairs $\left(u_{k}, v_{k}\right)$ are all distinct and arranged in increasing lexicographic order. (Such arrays correspond to matrices $A$ of zeros and ones.) Starting with empty tableaux $P$ and $Q$ as in (3.2), we perform the following steps for $k=1,2, \cdots, N$ (in this order):

A1*. INSERT* $^{*}\left(v_{k}\right)$ into the dual tableau $P$, determining values $s_{k}, t_{k}$ in step $\mathrm{I} 5^{*}$.

$\mathrm{A} 2^{*}$. Set $q_{s_{k} t_{k}} \leftarrow u_{k}$.

By Theorem $1^{*}$ and an argument like that of $\S 3$, this makes $Q$ a generalized Young tableau, while $P$ is a dual tableau. The inverse procedure consists of the following steps for $k=N, \cdots, 2,1$ (in this order):

B1*. Find $s_{k}, t_{k}$ such that $q_{s_{k} t_{k}}$ is the largest positive integer element of $Q$, where $t_{k}$ is as large as possible. Set $u_{k} \leftarrow q_{s_{k} t_{k}}$ and then set $v_{s_{k} t_{k}} \leftarrow \infty$.

B2*. DELETE* $\left(s_{k}, t_{k}\right)$ from tableau $P$, determining a value $x$ in step D5*; set $v_{k} \leftarrow x$.

By Theorem $1^{*}$ this procedure will produce a two-line array (5.2) of distinct pairs in increasing lexicographic order, when given any dual tableau $P$ together with a generalized Young tableau $Q$ of the same shape. Therefore we have a correspondence between such pairs of tableaux and zero-one matrices.

The graph-theoretic equivalent of this construction, corresponding to $\S 4$, is obtained by changing (4.2) to

$$
u \leqq u^{\prime} \text { and } v<v^{\prime} \text {. }
$$

This lack of symmetry makes it impossible to find a simple relation between the tableaux corresponding to a matrix and its transpose; in general the latter two pairs of tableaux can be quite different.

6. Further properties. Let us now concentrate momentarily on the $P$ tableau, independently of $Q$. By varying $Q$, we will in general find many arrays $A$ corresponding to the same $P$ tableau, and it is of interest to look for characteristic properties of such arrays.

TheOREM 5. Let $x, x^{\prime}, x^{\prime \prime}$ be positive integers and let $Y$ be a generalized Young tableau. If $x \leqq x^{\prime}<x^{\prime \prime}$ the sequence of operations

$$
\text { INSERT }\left(x^{\prime \prime}\right), \operatorname{INSERT}(x), \operatorname{INSERT}\left(x^{\prime}\right)
$$

has the same effect on $Y$ as the sequence

INSERT $(x)$, INSERT $\left(x^{\prime \prime}\right)$, INSERT $\left(x^{\prime}\right)$. 
If $x<x^{\prime} \leqq x^{\prime \prime}$, the sequence

INSERT $\left(x^{\prime}\right)$, INSERT $(x)$, INSERT $\left(x^{\prime \prime}\right)$

has the same effect as

INSERT $\left(x^{\prime}\right)$, INSERT $\left(x^{\prime \prime}\right)$, INSERT $(x)$.

Proof. Using notation like that in the proof of Theorem 1, we will show that the sequences in question have the same effect on the first row of $Y$, and that the corresponding elements $x_{2}, x_{2}^{\prime}, x_{2}^{\prime \prime}$ will (by induction) yield the same results on the remainder of $Y$. By convention let us say that INSERT $(\infty)$ is the null operation; when $x^{\prime \prime}=$ $\infty$ the theorem holds trivially.

It is not difficult to verify that if $x$ does not displace $x^{\prime \prime}$ in (6.1), then the elements $x_{2}, x_{2}^{\prime}, x_{2}^{\prime \prime}$ are identical for sequences (6.2) and (6.1), and $x_{2} \leqq x_{2}^{\prime}<x_{2}^{\prime \prime}$. On the other hand if $x$ does displace $x^{\prime \prime}$ in (6.1) then the first row of $Y$ in its original state had the form

$$
\cdots y y^{\prime} y^{\prime \prime} \cdots
$$

where $y \leqq x \leqq x^{\prime}<x^{\prime \prime}<y^{\prime} \leqq y^{\prime \prime}$. After (6.1), the first row of $Y$ becomes

$$
\cdots y x x^{\prime} \cdots
$$

and $x_{2}^{\prime \prime}=y^{\prime}, x_{2}=x^{\prime \prime}, x_{2}^{\prime}=y^{\prime \prime}$. If instead we use (6.2), the first row becomes

$$
\cdots y x x^{\prime} \cdots
$$

and $x_{2}=y^{\prime}, x_{2}^{\prime \prime}=y^{\prime \prime}, x_{2}^{\prime}=x^{\prime \prime}$. By induction (using the fact that (6.3) is equivalent to $(6.4)$ ),

INSERT $\left(y^{\prime}\right), \operatorname{INSERT}\left(x^{\prime \prime}\right), \operatorname{INSERT}\left(y^{\prime \prime}\right)$

has the same effect as

INSERT $\left(y^{\prime}\right)$, INSERT $\left(y^{\prime \prime}\right)$, INSERT $\left(x^{\prime \prime}\right)$.

hence (6.1) is equivalent to (6.2).

A similar but somewhat simpler proof shows that (6.3) is equivalent to $(6.4)$.

Note that, once the two-line array (3.1) has been put into lexicographic order, the $P$ tableau constructed in $\S 3$ is a function of the sequence $v_{1}, v_{2}, \cdots, v_{N}$ only. In general let $\left(v_{1}, v_{2}, \cdots, v_{n}\right)$ be any sequence of positive integers, and let $P\left(v_{1}, v_{2}, \cdots, v_{n}\right)$ be the generalized Young tableau obtained by starting with an empty tableau and suc- 
cessively performing the operations

INSERT $\left(v_{1}\right)$, INSERT $\left(v_{2}\right) \cdots$, INSERT $\left(v_{n}\right)$.

We will write

$$
\begin{aligned}
\left(v_{1}, \cdots, v_{n}\right) & \equiv\left(w_{1}, \cdots, w_{n}\right) \text { if and only if } \\
P\left(v_{1}, \cdots, v_{n}\right) & =P\left(w_{1}, \cdots, w_{n}\right) .
\end{aligned}
$$

According to Theorem 5,

$$
\left(v_{1}, \cdots, v_{n}\right) \equiv\left(v_{1}, \cdots, v_{k-1}, v_{k+1}, v_{k}, v_{k+2}, \cdots, v_{n}\right)
$$

if

$$
v_{k} \leqq v_{k+2}<v_{k+1} \text { or } v_{k}<v_{k-1} \leqq v_{k+1} .
$$

Symbolically, if $a<b<c$, we have

$$
a c b \equiv c a b, b a c \equiv b c a, a b a \equiv b a a, b a b \equiv b b a .
$$

Using these elementary transformations we can usually find several sequences equivalent to a given one; for example,

$$
\begin{aligned}
&(4,2,1,1,2,3) \equiv(4,1,2,1,2,3) \equiv(1,4,2,1,2,3) \\
& \equiv(1,4,2,2,1,3) \equiv(4,1,2,2,1,3) \equiv(4,1,2,2,3,1) \\
& \equiv(1,4,2,2,3,1) \equiv(1,2,4,2,3,1) \equiv(1,2,4,2,1,3) \\
& \equiv(1,2,2,4,3,1) .
\end{aligned}
$$

In fact, it is somewhat surprising that the elementary transformations of Theorem 4 precisely characterize those sequences which yield the same $P$ tableau:

Theorem 6. $P\left(v_{1}, \cdots, v_{n}\right)=P\left(w_{1}, \cdots, w_{n}\right)$ if and only if $\left(v_{1}, \cdots, v_{n}\right)$ can be transformed into $\left(w_{1}, \cdots, w_{n}\right)$ by a sequence of the elementary transformations (6.6).

Proof. Let $P\left(v_{1}, \cdots, v_{n}\right)$ be a generalized Young tableau $Y$ of shape $\left(p_{1}, \cdots, p_{m}\right)$; and let $\left(u_{1}, \cdots, u_{n}\right)=\left(y_{m 1}, \cdots, y_{m p_{m}}, \cdots, y_{21}, \cdots\right.$, $\left.y_{2 p_{2}}, y_{11}, \cdots, y_{1 p_{1}}\right)$. It is easy to show that $P\left(v_{1}, \cdots, v_{n}\right)=P\left(u_{1}, \cdots, u_{n}\right)$; hence in view of Theorem 5 it suffices to prove that $\left(v_{1}, \cdots, v_{n}\right)$ can be transformed into the "canonical" sequence $\left(u_{1}, \cdots, u_{n}\right)$ for the tableau $Y$ using only the operations (6.6). By induction on $n$, we need only prove the following statement: If $x$ is a positive integer, if $\left(u_{1}, \cdots, u_{n}\right)$ is the canonical sequence for a generalized Young tableau $Y$, and if $\left(u_{1}^{\prime}, \cdots, u_{n}^{\prime}, u_{n+1}^{\prime}\right)$ is the canonical sequence for $Y$ after the operation INSERT $(x)$ has been performed, then $\left(u_{1}, \cdots, u_{n}, x\right)$ can be transformed into $\left(u_{1}^{\prime}, \cdots, u_{n+1}^{\prime}\right)$ using the operations (6.6). 
This necessary condition is easily proved, for if we assume that

$$
y_{1} \leqq \cdots \leqq y_{j-1} \leqq x<y_{j} \leqq y_{j+1} \leqq \cdots \leqq y_{k}, 1 \leqq j \leqq k,
$$

we have

$$
\begin{aligned}
\left(y_{1}, \cdots, y_{k}, x\right) & \equiv \cdots \equiv\left(y_{1}, \cdots, y_{j-1}, y_{j}, x, y_{j+1}, \cdots, y_{k}\right) \\
& \equiv \cdots \equiv\left(y_{j}, y_{1}, \cdots, y_{j-1}, x, y_{j+1}, \cdots, y_{k}\right)
\end{aligned}
$$

by rules (6.6); hence we can simulate the insertion algorithm (since $y_{j}=x_{2}$, etc.).

CoRollary. If $P\left(v_{1}, \cdots, v_{m}\right)=P\left(w_{1}, \cdots, w_{m}\right)$ and $P\left(v_{m+1}, \cdots, v_{n}\right)=$ $P\left(w_{m \div 1}, \cdots, w_{n}\right)$, then

$$
P\left(v_{1}, \cdots, v_{m}, v_{m+1}, \cdots, v_{n}\right)=P\left(w_{1}, \cdots, w_{m}, w_{m+1}, \cdots, w_{n}\right) .
$$

This corollary can be expressed in terms of matrices as follows: We denote the $P$ tableau corresponding to matrix $A$ by $P(A)$. Let

$$
A=\left(\begin{array}{l}
A_{1} \\
A_{2}
\end{array}\right), \quad B=\left(\begin{array}{l}
B_{1} \\
B_{2}
\end{array}\right)
$$

be two $\left(m_{1} \div m_{2}\right) \times n$ matrices partitioned into $m_{1} \times n$ and $m_{2} \times n$ submatrices. If $P\left(A_{1}\right)=P\left(B_{2}\right)$ and $P\left(A_{2}\right)=P\left(B_{2}\right)$, then $P(A)=P(B)$. A similar statement holds for the $Q$ tableau, by taking transposes (cf. Theorem 2).

The corollary can also be used to define an associative binary operation on generalized Young tableaux if we let

$$
P\left(v_{1}, \cdots, v_{m}\right) P\left(v_{m \div 1}, \cdots, v_{n}\right)=P\left(v_{1}, \cdots, v_{n}\right) .
$$

Some very special cases of this associative operation were discovered by Schensted in his original paper [5]. Perhaps some further properties of the correspondence can be deduced from a deeper study of this "tableau algebra."

Similar properties can be developed for the dual correspondence of $\S 4$. If we interchange $<$ and $\leqq$ in Theorem 5 and change INSERT to INSERT*, we obtain Theorem $5^{*}$, which leads to a corresponding Theorem $6^{*}$ and its corollary in an analogous way. We can also show that the elementary transformations (6.6) allow us to build the dual tableau $P^{*}$ of a sequence by working on columns instead of rows during the insertion algorithm, and inserting the elements in the order $v_{n}, \cdots$, $v_{2}, v_{1}$. Therefore $P\left(v_{n}, \cdots, v_{1}\right)$ is the transpose of $P^{*}\left(v_{1}, \cdots, v_{n}\right)$; this interesting relation implies that the number of rows of $P\left(v_{1}, \cdots, v_{n}\right)$ is the length of the longest strictly decreasing subsequence of $\left(v_{1}, \cdots\right.$, $\left.v_{n}\right)$. (The latter result is due to Schensted [7].) The details underly- 
ing these observations are straightforward and left to the reader.

Let us conclude our remarks by deriving a further consequence of Theorem 6:

Theorem 7. Let $\pi(1), \cdots, \pi(m)$ be a permutation of the integers $\{1,2, \cdots, m\}$. There is a constructive one-to-one correspondence between the set of all nonnegative integer $m \times n$ matrices with row sums $\left(r_{1} \cdots, r_{m}\right)$ and those with row sums $\left(r_{\pi(1)}, \cdots, r_{\pi(m)}\right)$ such that, if $A$ corresponds to $B$, we have $P(A)=P(B)$.

Proof. Since any permutation is a product of adjacent transpositions, we may assume without loss of generality that $\pi$ merely interchanges $k$ and $k+1$. Let us partition

$$
A=\left(\begin{array}{l}
A_{1} \\
A_{2} \\
A_{3}
\end{array}\right),
$$

where $A_{1}$ has $k-1$ rows, $A_{2}$ has 2 rows, and $A_{3}$ has $m-k-1$ rows. Let the row sums of $k$ be $\left(r^{2}, s\right)$; it suffices to construct a $2 \times n$ matrix $B_{2}$ such that $P\left(A_{2}\right)=P\left(B_{2}\right)$ and such that the row sums of $B_{2}$ are $(s, r)$, since the corollary to Theorem 6 tells us that $P(A)=P(B)$ when

$$
B=\left(\begin{array}{c}
A_{1} \\
B_{2} \\
A_{3}
\end{array}\right) .
$$

The tableau $Q\left(A_{2}\right)$ has $r$ 1's and $(s-t) 2$ 's in its first row, and it has $t 2$ 's in its second row, for some $t \leqq \min (r, s)$. We now define $B_{2}$ by saying that $P\left(B_{2}\right)=P\left(A_{2}\right)$ and $Q\left(B_{2}\right)$ has $s$ 's and $(r-t) 2$ 's in its first row, $t 2$ 's in its second row. The correspondence of $\S 3$ determines a unique $B_{2}$ with this property, so the mapping $A_{2} \leftrightarrow B_{2}$ is reversible.

COROLLARY. Let $p_{1} \geqq p_{2} \geqq \cdots \geqq p_{m} \geqq 1$, and $p_{1}+p_{2}+\cdots+p_{m}=$ $N=r_{1}+r_{2}+\cdots+r_{m}$, where $r_{1}, r_{2}, \cdots$ are positive integers. Let $\pi(1), \cdots, \pi(m)$ be a permutation of the integers $\{1,2, \cdots, m\}$. There is a constructive one-to-one correspondence between the set of all generalized Young tableaux of shape $\left(p_{1}, \cdots, p_{m}\right)$ having $r_{1} 1$ 's $r_{2} 2$ 's, $\cdots$, $r_{m} m$ 's, and those tableaux of the same shape having $r_{\tau(1)} 1$ 's, $r_{\pi(2)}$ $2 ' s, \cdots, r_{\pi(m)} m$ 's.

Proof. Let $P$ be any fixed generalized Young tableau of shape $\left(p_{1}, \cdots, p_{m}\right)$, and consider the correspondence of Theorem 7 as $Q$ varies 
over all tableaux of the same shape, having $r_{i}$ occurrences of element $i$.

In other words, the number of ways to fill a shape with specified numbers of elements of different kinds is actually independent of the order of those elements.

7. Generating functions, Let $x=\left(x_{1}, \cdots, x_{n}\right)$ be a vector of formal variables, and let

$$
\{x ; p\}=\sum_{Y} \prod_{i=1}^{m} Y_{i 1} \cdots Y_{i p_{i}}
$$

summed over all generalized Young tableaux $Y$ of shape $p=\left(p_{1}, p_{2}, \cdots\right.$, $p_{m}$ ) that have been filled with the elements $x_{1}<x_{2}<\cdots<x_{n}$ each repeated any number of times. For example, we have

$$
\left\{x_{1}, x_{2}, x_{3} ; 2,1\right\}=x_{1}^{2} x_{2}+x_{1}^{2} x_{3}+x_{1} x_{2}^{2}+x_{1} x_{3}^{2}+x_{2}^{2} x_{3}+x_{2} x_{3}^{2}+2 x_{1} x_{2} x_{3}
$$

corresponding to the tableaux

$$
\begin{array}{llllllll}
11 & 11 & 12 & 13 & 22 & 23 & 12 & 13 \\
2 & 3 & 2 & 3 & 3 & 3 & 3 & 2
\end{array} .
$$

Dudley E. Littlewood [4, p. 191] has shown by group-theoretic means that $\{x ; p\}$ is a symmetric function of the $x$ 's, which is identical to a function studied by Jacobi, Schur, and others.

The two correspondences we have exhibited therefore provide a constructive proof of Littlewood's identities [3, Theorem V]

$$
\begin{aligned}
& \prod_{i=1}^{m} \prod_{j=1}^{n} \frac{1}{1-x_{i} y_{j}}=\sum_{p}\left\{x_{1}, \cdots, x_{m} ; p\right\}\left\{y_{1}, \cdots, y_{n} ; p\right\} \\
& \prod_{i=1}^{m} \prod_{j=1}^{n}\left(1+x_{i} y_{j}\right)=\sum_{p}\left\{x_{1}, \cdots, x_{m} ; p\right\}\left\{y_{1}, \cdots, y_{n} ; p^{T}\right\}
\end{aligned}
$$

summed over all shapes $p$, where $p^{T}$ denotes the transposed shape. The Jacobi-Trudi identity and the Naegelsbach-Kostka identity [4, pp. 88-89] can also be established combinatorially by means of our correspondences, as shown in [1].

Acknowledgments. I wish to thank Edward A. Bender for many stimulating discussions concerning the problems considered in this paper, and I also wish to thank Prof. Schützenberger for calling my attention to Schensted's construction and to reference [5]. 


\section{REFERENCES}

1. Edward A. Bender, and Donald E. Knuth, Enumeration of plane partitions, J. Combinatorial Theory (to appear)

2. Donald E. Knuth, The art of computer programming 1 (Reading, Mass.: AddisonWesley, 1968).

3. D. E. Littlewood, Some properties of S-functions, Proc. London Math. Soc. (2) 40 (1936), 40-70.

4. - The theory of group characters, Oxford, 1940.

5. G. de B. Robinson, On the representations of the symmetric group, Amer. J. Math. 60 (1938), 745-760; 69 (1947), 286-298; 70 (1948), 277-294.

6. Daniel E. Rutherford, Substitutional analysis (New York: Hafner, 1968).

7. C. Schensted, Longest increasing and decreasing subsequences, Canad. J. Math. 13 (1961), 179-191.

8. M. P. Schützenberger, Quelques remarques sur une construction de Schensted, Math. Scand. 12 (1963), 117-128.

Received May 14, 1969.

Institute for Defense Analyses 



\section{PACIFIC JOURNAL OF MATHEMATICS}

\section{EDITORS}

H. SAMELSON

Stanford University

Stanford, California 94305

\section{Richard Pierce}

University of Washington

Seattle, Washington 98105
J. DugundJI

Department of Mathematics

University of Southern California

Los Angeles, California 90007

RICHARD ARENS

University of California

Los Angeles, California 90024

\section{ASSOCIATE EDITORS}

\section{E. F. BeCKenBACH}

B. H. NeUmanN
K. YosHida

\section{SUPPORTING INSTITUTIONS}

\author{
UNIVERSITY OF BRITISH COLUMBIA \\ CALIFORNIA INSTITUTE OF TECHNOLOGY \\ UNIVERSITY OF CALIFORNIA \\ MONTANA STATE UNIVERSITY \\ UNIVERSITY OF NEVADA \\ NEW MEXICO STATE UNIVERSITY \\ OREGON STATE UNIVERSITY \\ UNIVERSITY OF OREGON \\ OSAKA UNIVERSITY \\ UNIVERSITY OF SOUTHERN CALIFORNIA
}

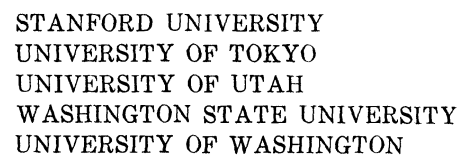

STANFORD UNIVERSITY UNIVERSITY OF TOKYO

UNIVERSITY OF UTAH

WASHINGTON STATE UNIVERSITY

UNIVERSITY OF WASHINGTON

The Supporting Institutions listed above contribute to the cost of publication of this Journal, but they are not owners or publishers and have no responsibility for its content or policies.

Mathematical papers intended for publication in the Pacific Journal of Mathematics should be in typed form or offset-reproduced, (not dittoed), double spaced with large margins. Underline Greek letters in red, German in green, and script in blue. The first paragraph or two must be capable of being used separately as a synopsis of the entire paper. The editorial "we" must not be used in the synopsis, and items of the bibliography should not be cited there unless absolutely necessary, in which case they must be identified by author and Journal, rather than by item number. Manuscripts, in duplicate if possible, may be sent to any one of the four editors. Please classify according to the scheme of Math. Rev. Index to Vol. 39. All other communications to the editors should be addressed to the managing editor, Richard Arens, University of California, Los Angeles, California, 90024.

50 reprints are provided free for each article; additional copies may be obtained at cost in multiples of 50 .

The Pacific Journal of Mathematics is published monthly. Effective with Volume 16 the price per volume (3 numbers) is $\$ 8.00$; single issues, $\$ 3.00$. Special price for current issues to individual faculty members of supporting institutions and to individual members of the American Mathematical Society: $\$ 4.00$ per volume; single issues $\$ 1.50$. Back numbers are available.

Subscriptions, orders for back numbers, and changes of address should be sent to Pacific Journal of Mathematics, 103 Highland Boulevard, Berkeley, California, 94708.

PUBLISHED BY PACIFIC JOURNAL OF MATHEMATICS, A NON-PROFIT CORPORATION

Printed at Kokusai Bunken Insatsusha (International Academic Printing Co., Ltd.), 7-17, Fujimi 2-chome, Chiyoda-ku, Tokyo, Japan. 


\section{Pacific Journal of Mathematics}

\section{Vol. 34, No. 3 \\ July, 1970}

Richard Hindman Bouldin, The peturbation of the singular spectrum

Hugh D. Brunk and Søren Glud Johansen, A generalized Radon-Nikodym derivative .

Henry Werner Davis, F. J. Murray and J. K. Weber, Families of $L_{p}$-spaces

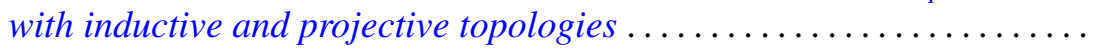

Esmond Ernest Devun, Special semigroups on the two-cell .

Murray Eisenberg and James Howard Hedlund, Expansive automorphisms

of Banach spaces ......................................

Frances F. Gulick, Actions of functions in Banach algebras.

Douglas Harris, Regular-closed spaces and proximities.

Norman Lloyd Johnson, Derivable semi-translation planes . .

Donald E. Knuth, Permutations, matrices, and generalized Young

tableaux..........................................

Herbert Frederick Kreimer, Jr., On the Galois theory of separable

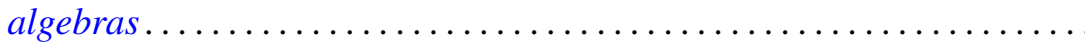

You-Feng Lin and David Alon Rose, Ascoli's theorem for spaces of

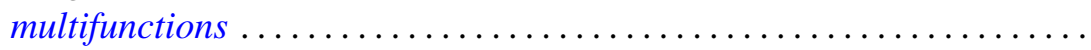

David London, Rearrangement inequalities involving convex functions . . . .

Louis Pigno, A multiplier theorem.

749

Helga Schirmer, Coincidences and fixed points of multifunctions into trees.

755

Richard A. Scoville, Some measure algebras on the integers .

Ralph Edwin Showalter, Local regularity of solutions of Sobolev-Galpern

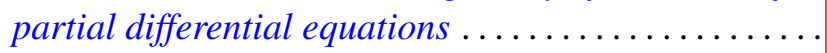

Allan John Sieradski, Twisted self-homotopy equivalences

John H. Smith, On S-units almost generated by S-units of subfields ...

803

Masamichi Takesaki, Algebraic equivalence of locally normal

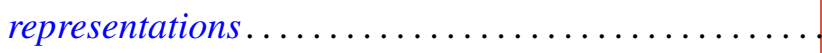

Joseph Earl Valentine, An analogue of Ptolemy's theorem and its converse in

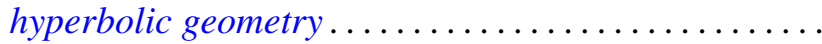

David Lawrence Winter, Solvability of certain p-solvable linear groups of finite order 